\title{
Determining the mechanism of LINE-1 ribonucleoprotein particle assembly and inhibition by nucleoside reverse transcriptase inhibitors.
}

\author{
Jocelyn C Newton*, Gerwald Jogl", John M Sedivy\# \\ "Graduate Program in Pathobiology and "Department of Molecular Biology, Cell Biology, and Biochemistry, \\ Brown University, Providence, RI 02912
}

Mobile genetic elements are sequences of DNA capable of altering their genomic location, resulting in host DNA damage and genomic instability. In humans, Long Interspersed Nuclear Element-1 (LINE-1) is the only autonomously replicating element, accounting for $17 \%$ of the genome ${ }^{1}$. Age-associated changes in the chromatin landscape and immune function are correlated with the transcriptional derepression of the LINE-1 element during cellular senescence. Two open reading frames produce a chaperone ORF1 protein and a catalytic ORF2 protein which assemble onto its native transcript to form a ribonucleoprotein (RNP) particle. The ORF2 protein consists of endonuclease, reverse transcriptase (RT), and nucleic acid binding domains whose functions are required for successful retrotransposition. After assembly, the LINE-1 RNP particle enters the nucleus and integrates into new genomic locations via a 'copy-and-paste' mechanism using its RNA-intermediate. Through comparative homology modeling ${ }^{2-3}$, various constructs isolating the functional LINE-1 RT domain were designed, expressed, and purified from Escherichia coli. Nucleoside reverse transcriptase inhibitors (NRTIs) originally developed for treating human immunodeficiency virus $(\mathrm{HIV})^{4}$ also inhibit LINE-1 RT activity. Therefore, we are using complementary techniques in biochemistry and structural biology to elucidate both the mechanisms of LINE-1 RNA binding and inhibition by NRTIs to explore the LINE-1 RT as a potential drug target in age-associated diseases.

\footnotetext{
${ }^{1}$ Lander, ES et al. Initial sequencing and analysis of the human genome. Nature 409:860-921 (2001).

${ }^{2}$ Song $Y$ et al. High resolution comparative modeling with RosettaCM. Structure (2013).

${ }^{3} \mathrm{Xu} \mathrm{D}$ et al. FFAS-3D: Improving fold recognition by including optimized structural features and template reranking. Bioinformatics (2013).

${ }^{4} \mathrm{Dia} L$ et al. Effect of reverse transcriptase inhibitors on LINE-1 and Ty1 reverse trancriptase activities and on LINE-1 retrotranposition. BMC Biochemistry 12(18):(2011).
} 